\title{
A novel Two-Dimensional LMS (TDLMS) using sub-sampling mask and step-size index for small target detection
}

\author{
Tae-Wuk Bae ${ }^{1 \mathrm{a})}$, Young-Choon Kim², Sang-Ho Ahn ${ }^{3}$, \\ and Kyu-Ik Sohng ${ }^{1}$ \\ ${ }^{1}$ School of Electrical Engineering and Computer Science, Kyungpook National \\ University, Buk-Gu, Daegu, 702-701, Korea \\ ${ }^{2}$ Dept. of Information and Communication Engineering, Youngdong University, \\ Korea \\ ${ }^{3}$ School of Electronic Telecommunication, Inje University, Korea \\ a) fiendcorps@knu.ac.kr
}

\begin{abstract}
In this paper, we propose a novel TDLMS filter using a sub-sampling mask and step-size index for small target detection in infra-red (IR) imagery. The concept of the proposed TDLMS is to utilize the sub-sampling mask of the weight-matrix having the structure reducing effects of the target pixels in order to predict the background exactly. And the nonlinear step size using the luminance rate (LR) and contrast rate (CR) is used and then background is predicted finally by using the two-dimensional Gaussian distance (TDGD). Experimental results show that the proposed method exhibits higher detection rates in comparison to the conventional TDLMS filter.
\end{abstract}

Keywords: small target detection, TDLMS filter, IR

Classification: Microwave and millimeter wave devices, circuits, and systems

\section{References}

[1] F. Zhang, C. Li, and L. Shi, "Detecting and tracking dim moving point target in IR image sequence," Infrared Physics \& Technology, vol. 46, no. 4, pp. 323-328, April 2005.

[2] M. M. Hadhoud and D. W. Thomas, "The two-dimensional adaptive LMS (TDLMS) algorithm," IEEE Trans. Circuits Syst., vol. 35, no. 5, pp. 485494, May 1998.

[3] T. Soni, J. R. Zeidler, and W. H. Ku, "Performance of 2-D adaptive prediction filters for detection of small objects in image data," IEEE Trans. Image Process., vol. 2, no. 3, pp. 327-340, July 1993.

[4] P. A. Ffrench, J. R. Zeidler, and W. H. Ku, "Enhanced detectability of small objects in correlated clutter using an improved 2-D adaptive lattice algorithm," IEEE Trans. Image Process., vol. 6, no. 3, pp. 383-397, March 1997. 
[5] Y. Cao, R. Liu, and J. Yang, "Small target detection using twodimensional least mean square (TDLMS) filter based on neighbor analysis," Int. J. Infrared Millim. Waves, vol. 29, no. 2, pp. 188-200, 2008.

\section{Introduction}

In Infrared Warning System (IRWS) and Infrared search and track (IRST), the essential problem is detection of ultra-dim, stationary, point-like targets with clutter in infrared (IR) images. The target appears as a dim point embedded in a heavy cluttered background due to the long distance at picture-taking of IR images [1]. Furthermore, aero-optic disturbances and air turbulence make the SNR of an IR image very low $(\mathrm{SNR}<3)$ in the real environments. So, the exact detection of the target location is very difficult task.

The TDLMS filter as prediction filter has been researched up to recently. Hadhoud et al. [2] proposed a two-dimensional LMS filter, an extension of the well-known LMS adaptive algorithm. Soni et al. [3] studied the performance of TDLMS filters as pre-whitening filer for the detection of small objects. Ffrench at al. [4] presents the small target detection using 2-D adaptive lattice algorithm. However, these methods have not performed well under low contrast and noisy images. Recently, Cao et al. [5] proposed the target detection method using TDLMS based on the neighborhood analysis. The background pixel values, predicted by the surrounding blocks, can be more exact but still include prediction errors in target region.

A novel TDLMS filter that can efficiently detect small targets is proposed. The proposed TDLMS filter utilizes the sub-sampling mask of the weight-matrix having the structure reducing effects of the target pixels and predicting the background pixels. The nonlinear step-size is controlled by the LR and the CR. The background prediction is finally performed by using the TDGD.

\section{Basic TDLMS Filter}

TDLMS predicts image pixels with the reference input image, $X$, with the $M \times M$ size. The weight-matrix $W_{j}$ is convolved with the image in regards to the reference input, resulting in the filter output given by

$$
Y(m, n)=\sum_{l=0}^{N-1} \sum_{k=0}^{N-1} W_{j}(l, k) X(m-l, n-k)
$$

where $Y$ is the predicted image and $j$ is the iteration number given by $j=$ $m M+n . W_{j}$ is the weight-matrix with $N \times N$ size at the $j$-th iteration. The set of integers $(l, k)$ of the weight-matrix coefficients, are called the region of support of the filter. The error signal $e_{j}$ at the $j$-th iteration is calculated as

$$
e_{j}=D(m, n)-Y(m, n)
$$


where $D(m, n)$ is a desired value of the image pixel. The error value is used to update the coefficients of the weight-matrix. The updated weightmatrix is equal to the present weight-matrix plus a change proportional to the negative gradient of the error power. This method will be used to determine an approximate solution for the updated weight-matrix. According to this technique, the coefficient adjustment equation of the two-dimensional weightmatrix is given as the following:

$$
W_{j+1}(l, k)=W_{j}(l, k)+\mu e_{j} X(m-l, n-k)
$$

where $\mu$ is the step-size to control the error signal.

\section{Proposed TDLMS Filter}

The basic TDLMS filter requires the modifications of weight-matrix and the step-size to apply to the small target detection system. And the proposed TDLMS is shown in Fig. 1.

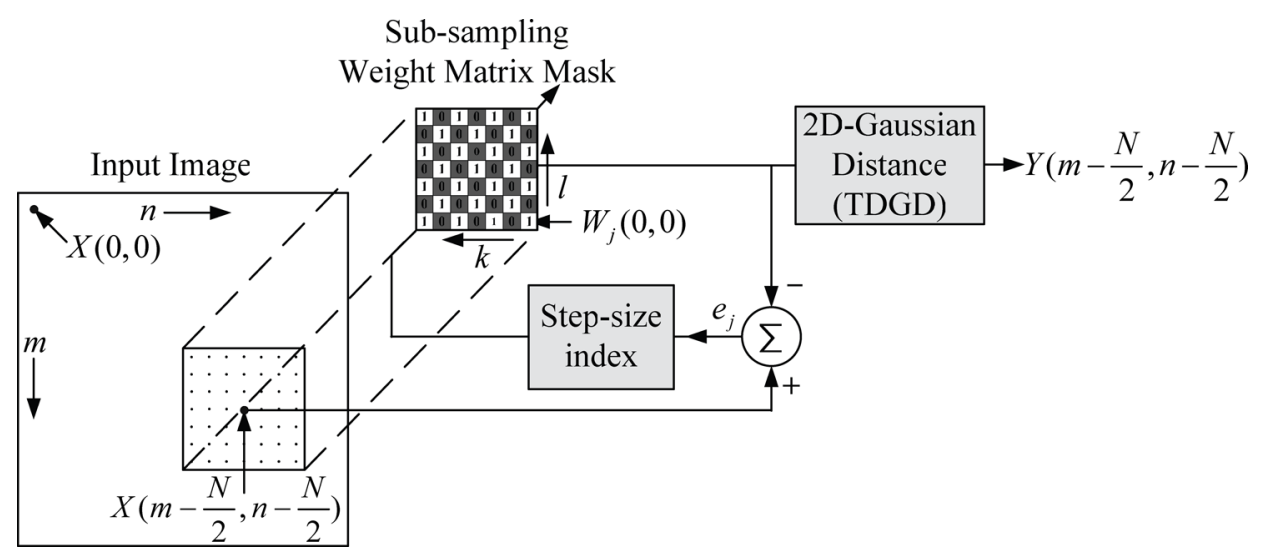

Fig. 1. The structure of the proposed TDLMS.

\subsection{Sub-sampling mask and TDGD}

The TDLMS filter predicts a background in regards to small target detection. Because the background in target region is covered by small targets, we use the sub-sampling mask of the weight-matrix, $M(l, k)$ to reduce the effect of target pixels in background prediction of the target region. Since actual target size is very small due to the long distance at picture-taking of IR images, the size of the weight-matrix is decided to $7 \times 7$. And we insert the TDGD map to give the larger weighting value for the weight-matrix coefficients in proportion to distance from center pixel. Because this distance map can give a greater weight to the outer pixels, it is useful in background prediction of target region. TDGD map, $G D(l, k)$, is given as the following:

$$
G D(l, k)=-e^{-\alpha\left((l-4)^{2}+(k-4)^{2}\right)}+1
$$

Where $l, k=1 \ldots . .7$ and $\alpha=0.5$. The parameter $\alpha$ determines the distribution of the Gaussian distance weight. 
Using the upper approach, the revised weight-matrix, $W M_{j}(l, k)$ and predicted pixel $Y$ is given as the following:

$$
\begin{aligned}
& W M_{j}(l, k)=W_{j}(l, k) M_{j}(l, k) \\
& Y(m, n)=\sum_{l=0}^{N-1} \sum_{k=0}^{N-1} W M_{j}(l, k) X(m-l, n-k) G D(l, k)
\end{aligned}
$$

Therefore, the revised weight adjustment algorithm is given by

$$
W M_{j+1}(l, k)=W M_{j}(l, k)+\mu_{j} e_{j} X(m-l, n-k) M_{j}(l, k)
$$

where $\mu_{j}$ is the nonlinear step-size controlled by step-size index in section 3.2 .

\subsection{Step-size index}

The proposed algorithm uses the step-size index using the random variable of the background region. During the transient state, such as a cloud edge and target region, a large $\mu$ is used. In order to prevent excessive steady-state parameter estimation errors, a smaller $\mu$ is used during steady state, such as a constant background.

Suppose that a random variable of the filter window is $x=\left\{x_{i} \mid i=\right.$ $1,2, \ldots, N\}$ and a random variable of the background region is $y=\left\{y_{i} \mid i=\right.$ $1,2, \ldots, N\}$ and the two random variable sets have the same distribution. $N=49$ is the number of the respective samples because the filter size is $7 \times 7$. The proposed step-size index, $\varphi$ is given as the following:

$$
\varphi=\frac{2 \bar{x} \bar{y}}{(\bar{x})^{2}+(\bar{y})^{2}} \cdot \frac{2 \sigma_{x} \sigma_{y}}{\left(\sigma_{x}^{2}+\sigma_{y}^{2}\right)}
$$

Where $\bar{x}, \sigma_{x}^{2}$ are the average and variance of $x$ and $\bar{y}, \sigma_{y}^{2}$ are the average and variance of $y$ and the dynamic range of $\varphi$ is $[0,1] . \varphi$ is modeled by the combination of the LR and CR. In eq. (7), the first component represents the LR of the two random variables and this means the similarity of the average luminance. In case of $\bar{x}=\bar{y}$, LR is 1 . In the second component, $\sigma_{x}^{2}$ and $\sigma_{y}^{2}$ represents the estimation of the contrast and means the similarity of CR between the filter window and the background. In case of $\sigma_{x}^{2}=\sigma_{y}^{2}$, CR is 1 . If $\varphi$ is close to 1 , that decides that the background and the filter window is the same region, $y_{i}=x_{i}$. On the other hand, if $\varphi$ is close to 0 , that decides that the filter window enter in the target region. Therefore we propose the nonlinear step-size using the step-size index as follows:

$$
\mu_{j}=\mu_{\min }+\frac{\mu_{\max }-\mu_{\min }}{1+e^{-\varphi}}
$$

where $\mu_{\max }$ and $\mu_{\min }$ are the maximum and minimum values of the step-size.

\subsection{The mapping function}

The subtracted image means the original image minus the predicted background image. Since the subtracted image has the positive and negative signs with a narrow range, it is mapped by following formula:

$$
F(m, n)=\log \left(\sigma^{2}(m, n)\right) \times e^{(S(m, n) / \tau)}
$$


where $F(m, n)$ and $S(m, n)$ are the mapping image and the subtracted image. $\sigma^{2}(m, n)$ is a variance of a $7 \times 7$ block in $S(m, n)$ and $\tau$ is the absolute difference value of the max and min values of the subtracted image. If eq. (9) has a minus value, these are mapped to zero. We regard pixels larger than threshold $T_{m}=0.9 F_{\max }$ as the candidate target centers, $F_{\max }$ is the maximum gray scale of the mapping image. In order to detect the target well and reduce the clutter, the $16 \times 16$ pixels around pixels of the candidate target center is processed by $3 \times 3$ mean filter and the residual region is processed by $3 \times 3$ min filter. Then the pixels more than 256 gray level is mapped to 256 gray level.

\section{Experiment results}

The computer simulations were run on 2 images (Image1, 2). We use $\mu_{\max }=$ $1.0 \times 10^{-7}$ and $\mu_{\text {min }}=1.0 \times 10^{-8}$. Image 1 has $320 \times 240$ size with low contrast and Image 2 has $360 \times 240$ size with the target in the cloud. Fig. 2 shows the detection results using the proposed TDLMS from 2 images. Column (a) shows original images containing a small target in and column (b) shows the prediction images estimated by the proposed TDLMS. Column (c) shows mapping images. The small target region was magnified the though the arrow and square in Column (a), (b), and (c). Image by mean and min filtering is shown in Column (d). We can show that the small target has distinct gray levels, compared with the background. Column (e) shows the step-size variation in 2 images. The blue color means that step-size is slow in the flat, constant region. The red color means that step-size is fast in the transient region as the cloud edge.

We computed the receiver operating characteristic curves (ROC) in order to measure the objective performance of the proposed method. A ROC curve plots the probability of the correct detection $P_{d}$ as a function of the number

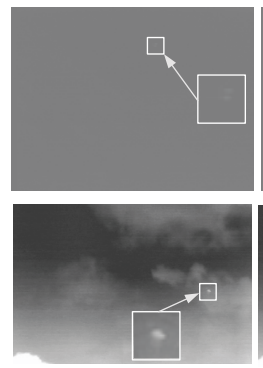

(a)

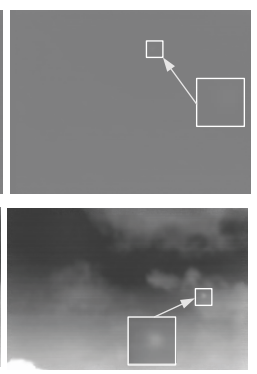

(b)

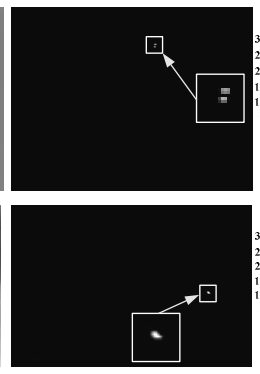

(c)

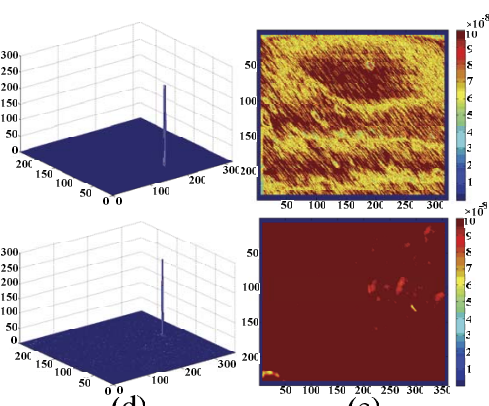

(e)

Fig. 2. Detection results of the small target about 2 images. Column (a) The original images, Column (b) The background prediction images, Column (c) The mapping images of the subtracted images, Column (d) Intensity plot after using mean, and min filtering by the classification process of the candidate target region, and Column (e) Step size plot of respective images. 


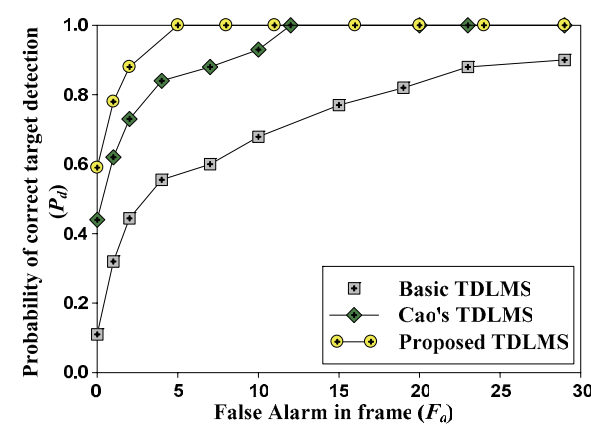

(a)

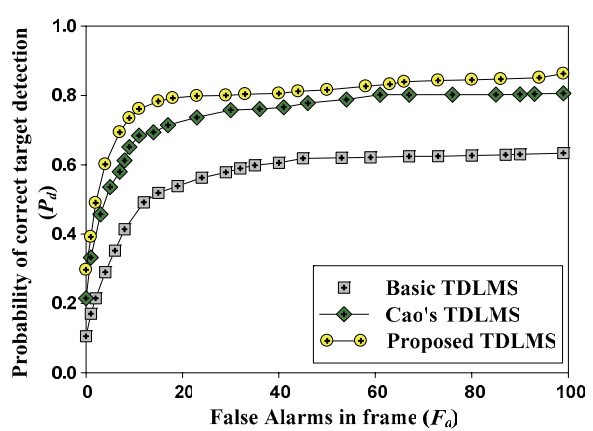

(b)

Fig. 3. The ROC curves obtained for the basic TDLMS, Cao's TDLMS, and the proposed TDLMS with: (a) Image1; (b) Image2.

$F_{a}$ of false alarms in frame, given by

$$
\begin{aligned}
P_{d} & =\frac{\text { number of detections in frame }}{\text { number of actual targets in frame }} \\
F_{a} & =\frac{\text { number of false detection in frame }}{\text { number of frame }}
\end{aligned}
$$

where number of frame of $F_{a}$ is 1 in target detection of the spatial processing. The detection criteria is provided as $w \times I_{\max }(0<w<1)$. $I_{\max }$ is the $\max$ intensity of the resulting image by the conventional TDLMS and the proposed TDLMS, and $w$ is the weighting factor controlling the ratio of targets and clutters. Fig. 3 shows the ROC curves of respective algorithms, using eq. (10) and (11). We can show that the proposed TDLMS has high target to clutter ratio in comparison to the previous TDLMSs. The basic TDLMS is simulated by $\mu=\left(\mu_{\max }+\mu_{\min }\right) / 2$. And the mapping function of Cao's TDLMS uses the fixed variance, but was adaptively modified in corresponding to the experiment images. The proposed TDLMS displays substantially better target detection performance in comparison to the conventional TDLMS at low false alarm rates.

\section{Conclusions}

In this paper, a novel TDLMS filter that can efficiently detect a small target is proposed in IR imagery. The proposed TDLMS filter utilizes the weightmatrix mask and step-size index having the structure reducing effects of the target pixels in order to predict exactly the background. The step size index utilizing the LR and the CR is used. Experimental results show that the proposed method exhibits higher detection rates and lower false alarm rates in comparison to the conventional TDLMS filter.

\section{Acknowledgments}

This work was supported by a basic research project of ADD (Agency for defense development) in Korea. 